\title{
NEVANLINNA-PICK INTERPOLATION FOR NON-COMMUTATIVE ANALYTIC TOEPLITZ ALGEBRAS
}

\author{
KENNETH R. DAVIDSON AND DAVID R. PITTS
}

The non-commutative analytic Toeplitz algebra is the WOT-closed algebra generated by the left regular representation of the free semigroup on $n$ generators. We obtain a distance formula to an arbitrary WOTclosed right ideal and thereby show that the quotient is completely isometrically isomorphic to the compression of the algebra to the orthogonal complement of the range of the ideal. This is used to obtain Nevanlinna-Pick type interpolation theorems.

In $[\mathbf{9}, \mathbf{1 0}]$, we studied the non-commutative analytic Toeplitz algebras $\mathfrak{L}_{n}$ associated to the free semi-group on $n$ generators. This is the WOT-closed algebra generated by the left regular representation. This algebra and its norm-closed version (the non-commutative disk algebra) were introduced by Popescu [19] in an abstract sense in connection with a non-commutative von Neumann inequality and further studied in several papers [17, 19, $\mathbf{2 0}, \mathbf{2 1}, \mathbf{2 3}$. We established a strong connection with the function theory on the ball $\mathbb{B}_{n}$ in $\mathbb{C}^{n}$ through our characterization [10] of the automorphism group. In particular, there is a natural homomorphism of $\mathfrak{L}_{n}$ into $H^{\infty}\left(\mathbb{B}_{n}\right)$. This leads to the natural question of which analytic functions are in the range of this map, and in particular, interpolation questions of the Nevanlinna-Pick type.

1991 Mathematics Subject Classification. 47D25.

First author partially supported by an NSERC grant and a Killam Research Fellowship.

Second author partially supported by an NSF grant. 
We let $\mathcal{F}_{n}$ denote the free semigroup on $n$ generators $z_{1}, \ldots, z_{n}$. (We allow $n=\infty$, but for convenience of notation, shall act as if $n$ is finite.) Form the Hilbert space $\mathcal{H}_{n}=$ $\ell^{2}\left(\mathcal{F}_{n}\right)$ with orthonormal basis $\xi_{w}$ for $w \in \mathcal{F}_{n}$. Then define the left regular representation by isometries $L_{v} \xi_{w}=\xi_{v w}$ for $v, w \in \mathcal{F}_{n}$. For simplification of notation, we write $L_{i}$ instead of $L_{z_{i}}$. The algebra $\mathfrak{L}_{n}$ is the WOT-closed algebra generated by $\left\{L_{1}, \ldots, L_{n}\right\}$. It is sometimes convenient to identify $\ell^{2}\left(\mathcal{F}_{n}\right)$ with the Fock space of $\mathbb{C}^{n}=\operatorname{span}\left\{e_{1}, \ldots, e_{n}\right\}$ by sending $\xi_{z_{i_{1}} \ldots z_{i_{k}}}$ to $e_{i_{1}} \otimes \cdots \otimes e_{i_{k}}$ for every word $w=z_{i_{1}} \ldots z_{i_{k}}$ in $\mathcal{F}_{n}$. This identifies $L_{i}$ with the left creation operator $L_{i} x=e_{i} \otimes x$.

In [3], Arias and Popescu use a Beurling type theorem from [17] to obtain the reflexivity of $\mathfrak{L}_{n}$. We discovered this Beurling theorem independently and used it to establish hyper-reflexivity [9, Theorem 2.9]. The Beurling theorem shows that cyclic invariant subspaces of $\mathfrak{L}_{n}$ correspond to the ranges of isometries in the commutant $\mathfrak{R}_{n}$, the right regular representation algebra. Moreover, every invariant subspace is the direct sum of cyclic invariant subspaces. Indeed an invariant subspace $\mathcal{M}$ is determined by a Wold decomposition by computing its wandering space $\mathcal{W}=\mathcal{M} \ominus \sum_{i=1}^{n} L_{i} \mathcal{M}$. Then $\mathcal{M}=\sum_{w \in \mathcal{F}_{n}} \oplus L_{w} \mathcal{W}$.

In addition to this description of invariant subspaces, we needed to know all the invariant subspaces of codimension one. These correspond to eigenvectors of the adjoint algebra $\mathfrak{L}_{n}^{*}$. They are classified [9, Theorem 2.6] by the points of the complex $n$-ball

$$
\mathbb{B}_{n}=\left\{\lambda \in \mathbb{C}^{n}:\|\lambda\|_{2}<1\right\}
$$

These vectors are given by the formula

$$
\nu_{\lambda}=\left(1-\|\lambda\|^{2}\right)^{1 / 2} \sum_{w \in \mathcal{F}_{n}} \overline{w(\lambda)} \xi_{w}=\left(1-\|\lambda\|^{2}\right)^{1 / 2}\left(I-\sum_{i=1}^{n} \overline{\lambda_{i}} L_{i}\right)^{-1} \xi_{1} .
$$

They yield the set of all WOT-continuous multiplicative linear functionals on $\mathfrak{L}_{n}$ by the formula

$$
\varphi_{\lambda}(A)=\left\langle A \nu_{\lambda}, \nu_{\lambda}\right\rangle
$$

Moreover the map taking $A$ to the function $\widehat{A}(\lambda)=\varphi_{\lambda}(A)$ is a completely contractive homomorphism of $\mathfrak{L}_{n}$ into $H^{\infty}\left(\mathbb{B}_{n}\right)$ [10, Theorem 3.3]. Finally, a calculation shows that for $\lambda_{1}, \lambda_{2} \in \mathbb{B}_{n}$,

$$
\left\langle\nu_{\lambda_{1}}, \nu_{\lambda_{2}}\right\rangle=\frac{\sqrt{1-\left|\lambda_{1}\right|^{2}} \sqrt{1-\left|\lambda_{2}\right|^{2}}}{1-\left\langle\lambda_{2}, \lambda_{1}\right\rangle}
$$


Further evidence of the strong connection with analytic functions is provided by the automorphism group. There is a natural map of $\operatorname{Aut}\left(\mathfrak{L}_{n}\right)$ onto the $\operatorname{group} \operatorname{Aut}\left(\mathbb{B}_{n}\right)$ of conformal automorphisms of the $n$-ball given by the dual action on the functionals $\varphi_{\lambda}[\mathbf{1 0}$, Theorem 4.1]. The kernel is the subgroup of automorphisms which are trivial modulo the WOT-closed commutator ideal $\overline{\mathfrak{C}}$. Moreover, the restriction of this map to the automorphisms which are unitarily implemented is an isomorphism. All automorphisms are automatically norm and WOT-continuous.

In the case of the algebra $H^{\infty}$ for the unit disk (which is the algebra $\mathfrak{L}_{1}$ ), Nevanlinna, Pick and Carathéodory considered interpolation questions of the following type: given points $\alpha_{j}$ and $c_{j}$ in $\mathbb{D}$ for $1 \leq j \leq k$, is there an analytic function $h \in H^{\infty}$ with $\|h\| \leq 1$ such that $h\left(\alpha_{j}\right)=c_{j}$ for $1 \leq j \leq k$. The answer to this and related questions is generally given by checking whether an affiliated matrix is positive semi-definite. We establish similar results in our context: given points $\alpha_{j}$ in $\mathbb{B}_{n}$ and matrices $C_{j}$ in $\mathfrak{M}_{p}$ for $1 \leq j \leq k$, is there an element $F$ in the unit ball of $\mathfrak{M}_{p}\left(\mathfrak{L}_{n}\right)$ such that $\widehat{F}\left(\alpha_{j}\right)=C_{j}$ for $1 \leq j \leq k$ ? We will determine necessary and sufficient conditions for this and related interpolation questions.

Our approach is modeled on that used by Sarason [25]. The kernel of the map taking $F$ to $\left(\widehat{F}\left(\alpha_{1}\right), \ldots, \widehat{F}\left(\alpha_{k}\right)\right)$ is the ideal of functions vanishing at these $k$ points. It was shown in [10, Theorem 2.1] that the map taking a WOT-closed right ideal to its range space is a complete lattice isomorphism onto the lattice of invariant subspaces of $\mathfrak{R}_{n}$, and that two-sided ideals correspond to subspaces which are also invariant for $\mathfrak{L}_{n}$. Thus we are led to computing the quotient map by an arbitrary ideal. This is shown to be completely isometric to the restriction to the complement of the associated range space. This spatial information will allow us to obtain the desired interpolation condition. It is a consequence of our methods that the map from $\mathfrak{L}_{n}$ into $H^{\infty}\left(\mathbb{B}_{n}\right)$ is not surjective.

The distance estimate is based on a refinement of the predual property $\mathbb{A}_{1}(1)$ established in [9, Theorem 2.10]. There it is shown that if $f$ is any weak-* continuous linear functional on $\mathfrak{L}_{n}$ with $\|f\|<1$, then there are vectors $\xi$ and $\zeta$ with $\|\xi\|\|\zeta\|<1$ such that $f(A)=\langle A \xi, \zeta\rangle$ for all $A \in \mathfrak{L}_{n}$. Here we need the corresponding property for $\mathfrak{L}_{n} \bar{\otimes} \mathcal{B}(\mathcal{K})$, the WOT-closed algebra of all operators with coefficients in $\mathfrak{L}_{n}$. This property is immediate from the proof for $\mathfrak{L}_{n}$. However it yields the generally stronger property $\mathbb{A}_{\aleph_{0}}$. 
In the last section, we relate our interpolation theorems to other similar results on reproducing kernel Hilbert spaces. The connection is the fact that symmetric Fock space is an analytic reproducing kernel Hilbert space [5]. This allows us to connect our results with results of Agler [2], McCullough [14] and Quiggin [24]. We also provide a new proof of a commutative dilation result of Popescu [22] and Arveson [5]. The dilation theory connects to interpolation via a version of the commutant lifting theorem in this context, which follows from Popescu's non-commutative version [18].

We take this opportunity to thank Vern Paulsen for several conversations about the content of this paper. In particular, this lead to the addition of the section on reproducing kernel Hilbert spaces.

After this paper was submitted for publication, we received a preprint from Arias and Popescu [4] which has significant overlap with our paper. Briefly, the principal overlapping parts are: $i$ ) the distance formula Theorem 2.1 is proved here for right ideals and in [4] for two-sided ideals; and $i i$ ) both papers contain Corollary 2.2, Theorem 3.2 and Theorem 3.3, although they are proved in [4] as a consequence of a commutant lifting theorem.

\section{PREDUAL PROPERTIES}

If $\mathfrak{A}$ is a WOT-closed operator algebra on a Hilbert space $\mathcal{H}$, let $\mathfrak{A} \bar{\otimes} \mathcal{B}(\mathcal{K})$ denote the WOT-closed algebra generated by the spatial tensor product of the two algebras. This consists of those operators acting on the Hilbert space $\mathcal{H} \otimes \mathcal{K}$ such that each "matrix coefficient" with respect to some (any) basis for $\mathcal{K}$ belong to $\mathfrak{A}$. The following result is proven in the same way as [9, Theorem 2.10] using the fact that the isometries $V \otimes I$ and $W \otimes I$ have orthogonal ranges and commute with $\mathfrak{A} \bar{\otimes} \mathcal{B}(\mathcal{K})$.

LEMMA 1.1. If $\mathfrak{A} \subseteq \mathcal{B}(\mathcal{H})$ is a wOT-closed algebra which commutes with two isometries $V$ and $W$ with orthogonal ranges, then the algebra $\mathfrak{A} \bar{\otimes} \mathcal{B}(\mathcal{K})$ has property $\mathbb{A}_{1}(1)$, namely given any weak-* continuous linear functional $\varphi$ and an $\varepsilon>0$, there are vectors $\eta$ and $\zeta$ belonging to $\mathcal{H} \otimes \mathcal{K}$ such that $\|\eta\|\|\zeta\|<\|\varphi\|+\varepsilon$ and $\varphi(A)=\langle A \eta, \zeta\rangle$ for all $A \in \mathfrak{A} \bar{\otimes} \mathcal{B}(\mathcal{K})$.

COROLLARY 1.2. $\mathfrak{L}_{n} \bar{\otimes} \mathcal{B}(\mathcal{K})$ has property $\mathbb{A}_{1}(1)$. 
This corollary is actually the precise form which we need, and for which we obtain good norm control. In the literature [6], the following variant is used. So we include it for completeness. We note that Bercovici [7] has established that any algebra commuting with two isometries with orthogonal ranges has the property $\mathcal{X}(0,1)$, which is closely related to the property $\mathbb{A}_{\aleph_{0}}$. He uses this to establish hyper-reflexivity for all such algebras with distance constant 3 (which is a much better estimate than the bound 51 which we obtained for $\mathfrak{L}_{n}$ in $\left.[9]\right)$.

An operator algebra has property $\mathbb{A}_{p}, 1 \leq p \leq \aleph_{0}$, if for every $p \times p$ matrix $\left[\varphi_{i j}\right]$ of weak-* continuous linear functionals, there are vectors $\eta_{i}$ and $\zeta_{j}$ for $1 \leq i, j \leq p$ such that

$$
\varphi_{i j}(A)=\left\langle A \eta_{j}, \zeta_{i}\right\rangle \quad \text { for all } \quad A \in \mathfrak{A} \text { and } 1 \leq i, j \leq p .
$$

It has property $\mathbb{A}_{p}(r)$ if one may choose $\left\|\eta_{j}\right\|<(r+\varepsilon)^{1 / 2}$ and $\|\zeta\|<(r+\varepsilon)^{1 / 2}$ provided that $\left\|\varphi_{i j}\right\| \leq 1$ for all $i$ and $j$.

COROLLARY 1.3. If $\mathfrak{A}$ is a WOT-closed algebra which commutes with two isometries $V$ and $W$ with orthogonal ranges, then it has properties $\mathbb{A}_{\aleph_{0}}$ and $\mathbb{A}_{p}\left(p^{2}\right)$ for $p \geq 1$.

Proof. If $p$ is finite and $\mathcal{K}_{p}$ is a Hilbert space of dimension $p$, the matrix $\Phi=\left[\varphi_{i j}\right]$ acts naturally as a linear functional on $\mathfrak{A} \otimes \mathcal{B}\left(\mathcal{K}_{p}\right)$ via the formula $\Phi(A)=\sum_{i, j} \varphi_{i j}\left(A_{i j}\right)$. Clearly, if $\left\|\varphi_{i j}\right\| \leq 1$ for all $i, j$, then $\|\Phi\| \leq p^{2}$. Using the fact that the tensor product has property $\mathbb{A}_{1}(1)$, we may find vectors $\eta=\left(\eta_{i}\right)_{i=1}^{p}$ and $\zeta=\left(\zeta_{i}\right)_{i=1}^{p}$ with $\|\eta\|<p+\varepsilon$ and $\|\zeta\|<p+\varepsilon$ such that

$$
\sum_{i, j=1}^{n} \varphi_{i j}\left(A_{i j}\right)=\Phi\left(\left[A_{i j}\right]\right)=\langle A \eta, \zeta\rangle=\sum_{i, j=1}^{n}\left\langle A_{i j} \eta_{j}, \zeta_{i}\right\rangle
$$

Hence we deduce that $\varphi_{i j}(A)=\left\langle A \eta_{j}, \zeta_{i}\right\rangle$ for $1 \leq i, j \leq p$. Thus $\mathfrak{A}$ has property $\mathbb{A}_{p}\left(p^{2}\right)$.

To prove property $\mathbb{A}_{\aleph_{0}}$, we use a simple rescaling device. Let $\Phi=\left[\varphi_{i j}\right]$ be an infinite matrix of weak-* continuous linear functionals on $\mathfrak{A}$. Let

$$
c_{k}=2^{k} \max \left\{1,\left\|\varphi_{i j}\right\|: \max \{i, j\}=k\right\}
$$

Then consider $\Psi=\left[c_{i}^{-1} c_{j}^{-1} \varphi_{i j}\right]$. It follows that

$$
\|\Psi\| \leq \sum_{i, j} c_{i}^{-1} c_{j}^{-1}\left\|\varphi_{i j}\right\| \leq \sum_{i, j} 2^{-i-j}=1 .
$$


Thus by Lemma 1.2 , there are vectors $\zeta=\left(\zeta_{i}\right)$ and $\eta=\left(\eta_{j}\right)$ for $i, j \geq 1$ such that $\Psi(A)=$ $\langle A \eta, \zeta\rangle$ for all $A \in \mathfrak{A} \bar{\otimes} \mathcal{B}(\mathcal{K})$. In particular,

$$
c_{i}^{-1} c_{j}^{-1} \varphi_{i j}(T)=\left\langle T \eta_{j}, \zeta_{i}\right\rangle \text { for all } T \in \mathfrak{A} .
$$

Thus the vectors $\eta_{j}^{\prime}=c_{j} \eta_{j}$ and $\zeta_{i}^{\prime}=c_{i} \zeta_{i}$ solve the interpolation problem.

COROLLARY 1.4. $\mathfrak{L}_{n}$ has properties $\mathbb{A}_{\aleph_{0}}$ and $\mathbb{A}_{p}\left(p^{2}\right)$ for $p \geq 1$.

\section{DISTANCE TO IDEALS}

As mentioned in the introduction, when $\mathfrak{J}$ is a wOT-closed right ideal of $\mathfrak{L}_{n}$, the range map

$$
\mu(\mathfrak{J})=\overline{\mathfrak{J} \mathcal{H}_{n}}=\overline{\mathfrak{J} \xi_{1}}
$$

is a complete lattice isomorphism onto Lat $\mathfrak{R}_{n}$ which carries the two-sided ideals onto Lat $\mathfrak{R}_{n} \cap$ Lat $\mathfrak{L}_{n}[\mathbf{1 0}$, Theorem 2.1]. The inverse is given by

$$
\iota(\mathcal{M})=\left\{J \in \mathfrak{L}_{n}: J \xi_{1} \in \mathcal{M}\right\} .
$$

Given such a right ideal $\mathfrak{J}$ with $\mathcal{M}=\mu(\mathfrak{J})$, the map which sends $A$ in $\mathfrak{L}_{n}$ to $P_{\mathcal{M}}^{\perp} A$ is completely contractive and annihilates $\mathfrak{J}$. Thus this map factors through the quotient $\mathfrak{L}_{n} / \mathfrak{J}$ with completely contractive maps. When $\mathfrak{J}$ is a two-sided ideal, $\mathcal{M}$ is invariant for $\mathfrak{L}_{n}$. In this case, $P_{\mathcal{M}}^{\perp} A=P_{\mathcal{M}}^{\perp} A P_{\mathcal{M}}^{\perp}$ is the compression to $\mathcal{M}^{\perp}$. Moreover, this compression map is a homomorphism. What we wish to establish is that this procedure determines the quotient completely isometrically.

THEOREM 2.1. Let $\mathfrak{J}$ be a WOT-closed right ideal in $\mathfrak{L}_{n} ;$ and let $\mathcal{M}=\mu(\mathfrak{J})=\overline{\mathfrak{J} \xi_{1}}$

denote its range. Then $\mathfrak{L}_{n} / \mathfrak{J}$ is completely isometric to $P_{\mathcal{M}}^{\perp} \mathfrak{L}_{n}$. That is, for every matrix $A=\left[A_{i j}\right]$ in $\mathfrak{M}_{p}\left(\mathfrak{L}_{n}\right), p \geq 1$,

$$
\operatorname{dist}\left(A, \mathfrak{M}_{p}(\mathfrak{J})\right)=\left\|\left(P_{\mathcal{M}}^{\perp} \otimes I_{p}\right) A\right\|
$$

Proof. In fact, our proof works just as well with $p=\aleph_{0}$, where $\mathfrak{M}_{p}\left(\mathfrak{L}_{n}\right)$ is used to mean $\mathfrak{L}_{n} \bar{\otimes} \mathcal{B}(\mathcal{K})$. Let $\varepsilon>0$ and suppose that $\operatorname{dist}\left(A, \mathfrak{M}_{p}(\mathfrak{J})\right)=1$. Choose a wot-continuous 
linear functional $\varphi$ on $\mathfrak{M}_{p}\left(\mathfrak{L}_{n}\right)$ which annihilates $\mathfrak{M}_{p}(\mathfrak{J})$ such that

$$
1-\varepsilon<|\varphi(A)| \leq\|\varphi\|=1
$$

By Corollary 1.2, there are vectors $\eta=\left(\eta_{i}\right)_{i=1}^{p}$ and $\zeta=\left(\zeta_{i}\right)_{i=1}^{p}$ in $\mathcal{H}_{n}^{(p)}$ such that $\varphi(T)=$ $\langle T \eta, \zeta\rangle$ for all $T \in \mathfrak{M}_{p}\left(\mathcal{L}_{n}\right)$ and $\|\eta\|\|\zeta\|<1+\varepsilon$.

Let

$$
\mathcal{N}=\overline{\sum_{i=1}^{p} \mathfrak{L}_{n} \eta_{i}} \quad \text { and } \quad \mathcal{N}_{0}=\overline{\sum_{i=1}^{p} \mathfrak{J} \eta_{i}}
$$

Clearly $\overline{\mathfrak{M}_{p}\left(\mathfrak{L}_{n}\right) \eta}=\mathcal{N}^{(p)}$ and $\overline{\mathfrak{M}_{p}(\mathfrak{J}) \eta}=\mathcal{N}_{0}^{(p)}$. Since $\mathcal{N}$ is an invariant subspace for $\mathfrak{L}_{n}$ which is generated by $p$ vectors, its wandering space has dimension $q \leq p$. Thus by $[\mathbf{9}$, Theorem 2.1], there are isometries $R_{j}$ in $\mathfrak{R}_{n}$ for $1 \leq j \leq q$ with pairwise orthogonal ranges such that

$$
\mathcal{N}=\sum_{j=1}^{q} \oplus R_{j} \mathcal{H}_{n}
$$

Observe that since $\mathfrak{J}$ is a right ideal, we have

$$
\mathcal{N}_{0}=\overline{\mathfrak{J} \mathcal{N}}=\sum_{j=1}^{q} \oplus R_{j} \mathcal{M}
$$

Therefore,

$$
R_{j}^{*} x \in \mathcal{M}^{\perp} \quad \text { for } \quad x \in \mathcal{N} \ominus \mathcal{N}_{0}
$$

Let $\left[\eta \zeta^{*}\right]$ be the functional on $\mathfrak{M}_{p}\left(\mathcal{B}\left(\mathcal{H}_{n}\right)\right)$ given by $A \mapsto(A \eta, \zeta)$. Since $\mathcal{N}^{(p)}$ is invariant for $\mathfrak{M}_{p}\left(\mathfrak{L}_{n}\right)$, we see that $\left[\eta \zeta^{*}\right]$ is unchanged on $\mathfrak{M}_{p}\left(\mathfrak{L}_{n}\right)$ if $\zeta$ is replaced by $\left(P_{\mathcal{N}} \otimes I_{p}\right) \zeta$. So we assume that this has been done. Also, since $\varphi$ annihilates $\mathfrak{M}_{p}(\mathfrak{J})$, it follows that $\zeta$ belongs to $\left(\mathcal{N} \ominus \mathcal{N}_{0}\right)^{(p)}$. Let

$$
\xi_{j}=\left(R_{j}^{*} \otimes I_{p}\right) \zeta \quad \text { and } \quad \nu_{j}=\left(R_{j}^{*} \otimes I_{p}\right) \eta \quad \text { for } \quad 1 \leq j \leq q .
$$

Equation (1) ensures that each $\xi_{i}$ belongs to $\mathcal{M}^{\perp(p)}$. Moreover,

$$
\zeta=\sum_{j=1}^{q} \oplus\left(R_{j} \otimes I_{p}\right) \xi_{j} .
$$

Let $\psi$ be the weak-*-continuous linear functional given by

$$
\psi(T)=\sum_{j=1}^{q}\left\langle T \nu_{j}, \xi_{j}\right\rangle .
$$


Now compute for $T \in \mathfrak{M}_{p}\left(\mathfrak{L}_{n}\right)$ :

$$
\begin{aligned}
\varphi(T) & =\langle T \eta, \zeta\rangle \\
& =\left\langle T \sum_{j=1}^{q} \oplus\left(R_{j} R_{j}^{*} \otimes I_{p}\right) \eta, \sum_{k=1}^{q} \oplus\left(R_{k} \otimes I_{p}\right) \xi_{k}\right\rangle \\
& =\sum_{j=1}^{q} \sum_{k=1}^{q}\left\langle\left(R_{k}^{*} \otimes I_{p}\right) T\left(R_{j} \otimes I_{p}\right)\left(R_{j}^{*} \otimes I_{p}\right) \eta, \xi_{k}\right\rangle \\
& =\sum_{j=1}^{q}\left\langle T\left(R_{j}^{*} \otimes I_{p}\right) \eta, \xi_{j}\right\rangle \\
& =\sum_{j=1}^{q}\left\langle T \nu_{j}, \xi_{j}\right\rangle=\psi(T) .
\end{aligned}
$$

Moreover,

$$
\begin{aligned}
\|\psi\| & \leq \sum_{j=1}^{q}\left\|\nu_{j}\right\|\left\|\xi_{j}\right\| \\
& \leq\left(\sum_{j=1}^{q}\left\|\nu_{j}\right\|\right)^{1 / 2}\left(\sum_{j=1}^{q}\left\|\xi_{j}\right\|\right)^{1 / 2} \\
& \leq\|\eta\|\|\zeta\|<1+\varepsilon .
\end{aligned}
$$

Since $\xi_{j}=\left(P_{\mathcal{M}}^{\perp} \otimes I_{p}\right) \xi_{j}$ for $1 \leq j \leq q$, it follows that

$$
|\psi(T)|=\left|\psi\left(\left(P_{\mathcal{M}}^{\perp} \otimes I_{p}\right) T\right)\right| \leq\|\psi\|\left\|\left(P_{\mathcal{M}}^{\perp} \otimes I_{p}\right) T\right\| .
$$

Therefore,

$$
\left\|\left(P_{\mathcal{M}}^{\perp} \otimes I_{p}\right) A\right\| \geq \frac{|\psi(A)|}{1+\varepsilon}=\frac{|\varphi(A)|}{1+\varepsilon}>\frac{1-\varepsilon}{1+\varepsilon} .
$$

As $\varepsilon>0$ was arbitrary, the result follows.

The desired conclusion for two-sided ideals is immediate:

COROLLARY 2.2. Let $\mathfrak{J}$ be a WOT-closed (two-sided) ideal in $\mathfrak{L}_{n}$; and let $\mathcal{M}=$ $\mu(\mathfrak{J})=\overline{\mathfrak{J} \xi_{1}}$ denote its range. Then $\mathfrak{L}_{n} / \mathfrak{J}$ is completely isometrically isomorphic to the compression of $\mathfrak{L}_{n}$ to $\mathcal{M}^{\perp}$.

Proof. By the previous theorem, the map taking $A+\mathfrak{J}$ to $P_{\mathcal{M}}^{\perp} A$ is a complete isometry. Since $\mathcal{M}$ is invariant for $\mathfrak{L}_{n}$ by [10, Theorem 2.1], $P_{\mathcal{M}}^{\perp} A=P_{\mathcal{M}}^{\perp} A P_{\mathcal{M}}^{\perp}$. Thus 
compression to $\mathcal{M}^{\perp}$ is also a complete isometry. The semi-invariance of $\mathcal{M}^{\perp}$ implies that the compression to $\mathcal{M}^{\perp}$ is a homomorphism.

As an application of the ideas, consider the Gelfand map taking $\mathfrak{L}_{n}$ into $H^{\infty}\left(\mathbb{B}_{n}\right)$ by sending $A$ to the function $\widehat{A}(\lambda)=\varphi_{\lambda}(A)$. By [10, Theorem 3.3], the kernel is precisely $\overline{\mathfrak{C}}$, the WOT-closed commutator ideal. By [10, Proposition 2.4],

$$
\mu(\overline{\mathfrak{C}})^{\perp}=\operatorname{span}\left\{\nu_{\lambda}: \lambda \in \mathbb{B}_{n}\right\}=: \mathcal{H}_{n}^{s}
$$

where $\mathcal{H}_{n}^{s}$ denotes the symmetric Fock space spanned by the tensors which are invariant under permutations. Hence we obtain that:

COROLLARY 2.3. The quotient $\mathfrak{L}_{n} / \overline{\mathfrak{C}}$ of $\mathfrak{L}_{n}$ by the WOT-closed commutator ideal is completely isometrically isomorphic to its compression to $\mathcal{H}_{n}^{s}$.

One immediately wonders whether this quotient is $H^{\infty}\left(\mathbb{B}_{n}\right)$. However it is not. The proof of this fact does not require the above distance estimate, only the easy direction. This argument is closely related to [5, Theorem 3.3], although it was found independently.

THEOREM 2.4. The quotient map of $\mathfrak{L}_{n}$ into $H^{\infty}$ given by sending $A$ in $\mathfrak{L}_{n}$ to $\widehat{A}$ is not surjective.

Proof. It suffices to show that the map from $\mathfrak{L}_{n} / \overline{\mathfrak{C}}$ to $H^{\infty}$ is not bounded below. To this end, consider the elements

$$
A_{k}=\sum_{w \in \mathcal{P}_{k}} L_{w}
$$

where $\mathcal{P}_{k}$ consists of all permutations of the word $z_{1}^{k} z_{2}^{k}$. Since there are $\left(\begin{array}{c}2 k \\ k\end{array}\right)$ such words and the corresponding isometries have orthogonal ranges, we see that $\left\|A_{k}\right\|=\left(\begin{array}{c}2 k \\ k\end{array}\right)^{1 / 2}$. Moreover, since $A_{k} \xi_{1}$ is a symmetric word and thus is orthogonal to the range $\mathcal{M}$ of the commutator ideal $\overline{\mathfrak{C}}$, it follows from the previous theorem (or by a simple bare-hands argument) that

$$
\left\|A_{k}\right\| \geq \operatorname{dist}\left(A_{k}, \overline{\mathfrak{C}}\right) \geq\left\|A_{k} \xi_{1}\right\|=\left(\begin{array}{c}
2 k \\
k
\end{array}\right)^{1 / 2}=\left\|A_{k}\right\| .
$$

Now $\widehat{A}_{k}=\left(\begin{array}{c}2 k \\ k\end{array}\right) \lambda_{1}^{k} \lambda_{2}^{k}$. It is easy to check that $\left\|\lambda_{1} \lambda_{2}\right\|_{\infty}=1 / 2$, so

$$
\left\|\widehat{A}_{k}\right\|_{\infty}=2^{-k}\left(\begin{array}{c}
2 k \\
k
\end{array}\right) \text {. }
$$


Estimating the ratio using Stirling's formula, we see that

$$
\begin{aligned}
\frac{\left\|\widehat{A}_{k}\right\|_{\infty}}{\operatorname{dist}\left(A_{k}, \overline{\mathfrak{C}}\right)} & =2^{-k}\left(\begin{array}{c}
2 k \\
k
\end{array}\right)^{1 / 2}=\frac{((2 k) !)^{1 / 2}}{2^{k} k !} \\
& \approx \frac{\left(\sqrt{2 \pi}(2 k)^{2 k+\frac{1}{2}} e^{-2 k}\right)^{1 / 2}}{2^{k} \sqrt{2 \pi} k^{k+\frac{1}{2}} e^{-k}}=(\pi k)^{-1 / 4} .
\end{aligned}
$$

\section{NEVANLINNA-PICK INTERPOLATION}

Nevanlinna and Pick considered the problem: given points $\alpha_{j}$ and $c_{j}$ in $\mathbb{D}$ for $1 \leq$ $j \leq k$, is there an analytic function $h \in H^{\infty}$ with $\|h\| \leq 1$ such that $h\left(\alpha_{j}\right)=c_{j}$ for $1 \leq j \leq k$. Carathéodory considered a related question: given a polynomial $p(z)=\sum_{j=0}^{k-1} a_{j} z^{j}$, is there a function in $H^{\infty}$ with $\|h\| \leq 1$ such that $h(z)=p(z)+\mathcal{O}\left(z^{k}\right)$. Both of these problems were solved classically. However Sarason [25] was able to reformulate these problems in an operator-theoretic context and provide new proofs based on a precursor of the commutant lifting theorem of Sz. Nagy and Foiaş [26]. This approach makes it almost as easy to deal with matrix interpolation questions. For an extensive treatment of this approach, see [11].

In this section we will use the distance estimate in Theorem 2.1 to obtain our interpolation results. Interestingly, Theorems 3.3 and 3.2 use Theorem 2.1 applied to twosided ideals, while Theorem 3.6 uses the distance estimate applied to a certain right ideal.

We first consider the general matrix Nevanlinna-Pick problem. Before stating the theorem, we require a simple lemma.

LEMMA 3.1. Given $k$ distinct points $\alpha_{j}$ in $\mathbb{B}_{n}$ and matrices $C_{j}$ in $\mathfrak{M}_{p}$ for $1 \leq$ $j \leq k$, there is an element $F$ in $\mathfrak{M}_{p} \mathfrak{L}_{n}$ such that $\widehat{F}\left(\alpha_{i}\right)=C_{i}$ for $1 \leq j \leq k$.

Proof. Let

$$
\mathfrak{J}=\bigcap_{i=1}^{n} \operatorname{ker}\left(\varphi_{\alpha_{j}}\right) \quad \text { and } \quad \mathfrak{J}_{j}=\bigcap_{\substack{i=1 \\ i \neq j}}^{n} \operatorname{ker} \varphi_{\alpha_{i}} \quad \text { for } \quad 1 \leq j \leq k .
$$

We first observe that $\mathfrak{J}$ is properly contained in $\mathfrak{J}_{j}$. This can be seen as follows. Since $\mu\left(\operatorname{ker}\left(\varphi_{j}\right)\right)^{\perp}=\operatorname{span}\left\{\nu_{\alpha_{j}}\right\}$, Theorem 2.1 of $[\mathbf{1 0}]$ shows that $\mu(\mathfrak{J})^{\perp}=\operatorname{span}\left\{\nu_{\alpha_{1}}, \ldots, \nu_{\alpha_{k}}\right\}$ and $\mu\left(\mathfrak{J}_{j}\right)^{\perp}=\operatorname{span}\left\{\nu_{\alpha_{i}}: i \neq j\right\}$. Since the $\nu_{\alpha_{j}}$ 's are linearly independent, $\mu(\mathfrak{J}) \neq \mu\left(\mathfrak{J}_{j}\right)$ and the 
observation follows. Thus we may choose $H_{j} \in \mathfrak{J}_{j} \backslash \mathfrak{J}$ so that $\widehat{H}_{j}\left(\alpha_{i}\right)=\delta_{i j}$. Now note that $F=\sum_{i=1}^{k} H_{i} \otimes C_{i}$ does the job.

THEOREM 3.2. Given $k$ distinct points $\alpha_{j}$ in $\mathbb{B}_{n}$ and matrices $C_{j}$ in $\mathfrak{M}_{p}$ for $1 \leq j \leq k$, there is an element $F$ in the unit ball of $\mathfrak{M}_{p}\left(\mathfrak{L}_{n}\right)$ such that $\widehat{F}\left(\alpha_{j}\right)=C_{j}$ for $1 \leq j \leq k$ if and only if the matrix

$$
\left[\frac{I_{p}-C_{i} C_{j}^{*}}{1-\left\langle\alpha_{i}, \alpha_{j}\right\rangle}\right]_{k \times k}
$$

is positive semidefinite.

Proof. Let $\mathfrak{J}=\bigcap_{j=1}^{k} \operatorname{ker} \varphi_{\alpha_{j}}$ and let $\mathcal{M}$ be the range space of $\mathfrak{J}$. Since $\mathfrak{J}$ is a two-sided ideal of $\mathfrak{L}_{n}, \mathcal{M}^{\perp}$ belongs to $\operatorname{Lat}\left(\mathfrak{L}_{n}^{*}\right)$, a fact we shall use shortly. It is clear that if we ignore the norm condition, that any two solutions to the interpolation problem differ by an element of $\mathfrak{M}_{p}(\mathfrak{J})$. Thus let $F$ be any element of $\mathfrak{M}_{p}\left(\mathfrak{L}_{n}\right)$ such that $\widehat{F}\left(\alpha_{j}\right)=C_{j}$ for $1 \leq j \leq k$. Then the norm condition is satisfied exactly when $\operatorname{dist}\left(F, \mathfrak{M}_{p}(\mathfrak{J})\right) \leq 1$.

By Corollary 2.2, this distance is computed as

$$
\operatorname{dist}\left(F, \mathfrak{M}_{p}(\mathfrak{J})\right)=\left\|\left(P_{\mathcal{M}}^{\perp} \otimes I_{p}\right) F\left(P_{\mathcal{M}}^{\perp} \otimes I_{p}\right)\right\|
$$

This is at most one if and only if

$$
\begin{aligned}
0 & \leq P_{\mathcal{M}}^{\perp} \otimes I_{p}-\left(P_{\mathcal{M}}^{\perp} \otimes I_{p}\right) F\left(P_{\mathcal{M}}^{\perp} \otimes I_{p}\right) F^{*}\left(P_{\mathcal{M}}^{\perp} \otimes I_{p}\right) \\
& =\left(P_{\mathcal{M}}^{\perp} \otimes I_{p}\right)\left(I-F F^{*}\right)\left(P_{\mathcal{M}}^{\perp} \otimes I_{p}\right),
\end{aligned}
$$

because $\mathcal{M}^{\perp} \in \operatorname{Lat}\left(\mathfrak{L}_{n}^{*}\right)$. Since $\left\{\nu_{\alpha_{j}}: 1 \leq j \leq k\right\}$ spans $\mathcal{M}^{\perp}$, a basis for $\mathcal{M} \otimes \mathbb{C}^{p}$ is given by the set $\nu_{\alpha_{j}} \otimes e_{r}$, where $e_{r}, 1 \leq r \leq p$, is an orthonormal basis for $\mathbb{C}^{p}$. Thus the norm condition is equivalent to

$$
0 \leq\left[\left\langle\left(I-F F^{*}\right) \nu_{\alpha_{i}} \otimes e_{r}, \nu_{\alpha_{j}} \otimes e_{s}\right\rangle\right] .
$$

Write $F=\left[F_{r s}\right]$. Then

$$
\begin{aligned}
& {\left[\left\langle\left(I-F F^{*}\right) \nu_{\alpha_{i}} \otimes e_{r}, \nu_{\alpha_{j}} \otimes e_{s}\right\rangle\right]_{k p \times k p}} \\
& =\left[\left\langle\nu_{\alpha_{i}}, \nu_{\alpha_{j}}\right\rangle \delta_{r s}\right]_{k p \times k p}-\left[\left\langle F^{*}\left(\nu_{\alpha_{i}} \otimes e_{r}\right), F^{*}\left(\nu_{\alpha_{j}} \otimes e_{s}\right)\right\rangle\right]_{k p \times k p} \\
& =\left[\left\langle\nu_{\alpha_{i}}, \nu_{\alpha_{j}}\right\rangle \delta_{r s}\right]_{k p \times k p}-\sum_{t=1}^{p}\left[\left\langle F_{r t}^{*} \nu_{\alpha_{i}}, F_{s t}^{*} \nu_{\alpha_{j}}\right\rangle\right]_{k p \times k p}
\end{aligned}
$$




$$
\begin{aligned}
& =\left[\left\langle\nu_{\alpha_{i}}, \nu_{\alpha_{j}}\right\rangle \delta_{r s}\right]_{k p \times k p}-\sum_{t=1}^{p}\left[\overline{\widehat{F}_{r t}\left(\alpha_{i}\right)} \widehat{F}_{s t}\left(\alpha_{j}\right)\left\langle\nu_{\alpha_{i}}, \nu_{\alpha_{j}}\right\rangle\right]_{k p \times k p} \\
& =\left[\left\langle\nu_{\alpha_{i}}, \nu_{\alpha_{j}}\right\rangle\left(I_{p}-\widehat{F}\left(\alpha_{j}\right) \overline{\widehat{F}\left(\alpha_{i}\right)}\right)^{t}\right]_{k \times k} \\
& =\left[\frac{\left(1-\left\|\alpha_{i}\right\|\right)^{1 / 2}\left(1-\left\|\alpha_{j}\right\|\right)^{1 / 2}\left(I_{p}-C_{j} C_{i}^{*}\right)^{t}}{1-\left\langle\alpha_{j}, \alpha_{i}\right\rangle}\right]_{k \times k} \\
& =\operatorname{diag}\left[\left(1-\left\|\alpha_{i}\right\|\right)^{1 / 2}\right]\left[\frac{\left(I_{p}-C_{j} C_{i}^{*}\right)^{t}}{1-\left\langle\alpha_{j}, \alpha_{i}\right\rangle}\right]_{k \times k} \operatorname{diag}\left[\left(1-\left\|\alpha_{j}\right\|\right)^{1 / 2}\right]
\end{aligned}
$$

Clearly this is positive if and only if the transposed matrix

$$
\left[\frac{I_{p}-C_{i} C_{j}^{*}}{1-\left\langle\alpha_{i}, \alpha_{j}\right\rangle}\right]_{k \times k} \geq 0 .
$$

It follows from Theorem 2.4 that the unit ball of $\mathfrak{L}_{n}$ cannot interpolate every $H^{\infty}$ function of norm one. The answer to which functions can be interpolated is our next example of a non-commutative Nevanlinna-Pick type of result.

THEOREM 3.3. Given an element $h \in H^{\infty}\left(\mathbb{B}_{n}\right)$, there is an operator $A \in \mathfrak{L}_{n}$ with $\|A\| \leq 1$ and $\widehat{A}=h$ if and only if the matrices

$$
\left[\frac{1-h\left(z_{i}\right) \overline{h\left(z_{j}\right)}}{1-\left\langle z_{i}, z_{j}\right\rangle}\right]
$$

are positive for all finite subsets $\left\{z_{j}: 1 \leq j \leq k\right\}$ of $\mathbb{B}_{n}$.

Proof. Let $A \in \mathfrak{L}_{n}$ be given, and let $h=\widehat{A}$. Given finitely many distinct points $z_{1}, \ldots, z_{k}$ in $\mathbb{B}_{n}$, we have $\widehat{A}\left(z_{j}\right)=h\left(z_{j}\right)$, so that Theorem 3.2 shows that the ma$\operatorname{trix}\left[\frac{1-h\left(z_{i}\right) \overline{h\left(z_{j}\right)}}{1-\left\langle z_{i}, z_{j}\right\rangle}\right]$ is positive.

Conversely, suppose $h \in H^{\infty}(\mathbb{B})$ is such that the matrices

$$
\left[\frac{1-h\left(z_{i}\right) \overline{h\left(z_{j}\right)}}{1-\left\langle z_{i}, z_{j}\right\rangle}\right]
$$

are positive for all finite subsets $\left\{z_{j}: 1 \leq j \leq k\right\}$ of $\mathbb{B}_{n}$. Let $\Delta$ be the set of all finite subsets of $\mathbb{B}_{n}$. For any finite set $\delta \in \Delta$, Theorem 3.2 shows

$$
W_{\delta}:=\left\{A \in \mathfrak{L}_{n}:\|A\| \leq 1 \text { and } \widehat{A}(\lambda)=h(\lambda) \text { for all } \lambda \in \delta\right\} \neq \emptyset
$$


Each $\varphi_{\lambda}$ is WOT-continuous, so $W_{\delta}$ is WOT-closed and hence is WOT-compact. Since the family $\left\{W_{\delta}: \delta \in \Delta\right\}$ has the finite intersection property, $\bigcap_{\delta \in \Delta} W_{\delta} \neq \emptyset$. Choosing $A$ in this intersection, we find $\widehat{A}(\lambda)=h(\lambda)$ for all $\lambda \in \mathbb{B}_{n}$.

Given $h \in H^{\infty}$ such that $h=\widehat{C}$ for some $C \in \mathfrak{L}_{n}$, Vern Paulsen has asked whether one can guess the element $A$ in $\mathfrak{L}_{n}$ with smallest norm satisfing $\widehat{A}=h$ by simply considering the "symmetrized" element of $\mathfrak{L}_{n}$. The following example shows the answer is no.

EXAMPLE 3.4. Consider the function $h(\lambda)=\lambda_{1}+\lambda_{1} \lambda_{2}$. The canonical choice for a lifting would be $A=L_{1}+\left(L_{1} L_{2}+L_{2} L_{1}\right) / 2$. However, we shall show that $B=L_{1}+L_{2} L_{1}$ is a lifting with smaller norm. Indeed, $B$ is the sum of two isometries with orthogonal ranges. So $\|B\|=\sqrt{2}$. Write $A=L_{1}\left(I+L_{2} / 2\right)+L_{2} L_{1} / 2$. These two terms have orthogonal ranges, and the latter $L_{2} L_{1} / 2$ is half an isometry. Thus

$$
\begin{aligned}
\|A\|^{2} & =\left\|I+L_{2} / 2\right\|^{2}+\left\|L_{2} L_{1} / 2\right\|^{2} \\
& =\left\|1+\lambda_{2} / 2\right\|_{\infty}^{2}+\frac{1}{4}=\left(\frac{3}{2}\right)^{2}+\frac{1}{4}=\frac{5}{2} .
\end{aligned}
$$

Indeed, a similar analysis shows that if $A_{t}=L_{1}+t L_{1} L_{2}+(1-t) L_{2} L_{1}$, then

$$
\left\|A_{t}\right\|=\sqrt{2+2|t|^{2}}
$$

The minimum clearly is attained at $t=0$.

Even the choice of $B$ is not optimal-higher order terms are needed. To see this, it suffices to show that $d=\operatorname{dist}(A, \overline{\mathfrak{C}})<\sqrt{2}$. By our distance formula, this is just $\left\|P_{\mathcal{M}} A\right\|=$ $\left\|\left.A^{*}\right|_{\mathcal{M}}\right\|$ where $\mathcal{M}=\overline{\mathfrak{C H}}_{2}^{\perp}=\mathcal{H}_{2}^{s}$. This space decomposes as a direct sum of the subspaces $\mathcal{S}_{k}=P_{\mathcal{M}} \operatorname{span}\left\{\xi_{w}: w(\lambda)=\lambda_{1}^{k} \lambda_{2}^{s}, s \geq 0\right\}$. Moreover, $P_{\mathcal{M}} A$ maps $\mathcal{S}_{k}$ into $\mathcal{S}_{k+1}$ for $k \geq 0$. The reader wishing to carry out the calculation should read the last section which makes it easy to compute that the compressions $S_{i}$ of $L_{i}$ to $\mathcal{M}$ are weighted shifts. It follows that the norm is equal to the norm of $\left.S_{1}\left(I+S_{2}\right)\right|_{\mathcal{S}_{0}}$, which yields $d^{2}=\|X\|$ where $X$ is the compact self-adjoint tridiagonal operator $X=\left[x_{i j}\right]$ given by $x_{k k}=\frac{1}{k}+\frac{1}{k+1}$ and $x_{k, k+1}=x_{k+1, k}=\frac{1}{k}$. It can be shown directly that $2 I-X$ is strictly positive, establishing the strict inequality.

It is clear that combining the matrix technique of the proof of Theorem 3.2 with the argument of Theorem 3.3, we obtain a matrix version of global interpolation: 
COROLLARY 3.5. Given an element $H \in \mathfrak{M}_{p}\left(H^{\infty}\left(\mathbb{B}_{n}\right)\right)$, there is an operator $A \in \mathfrak{M}_{p}\left(\mathfrak{L}_{n}\right)$ with $\|A\| \leq 1$ and $\widehat{A}=H$ if and only if the matrices

$$
\left[\frac{I_{p}-H\left(\alpha_{i}\right) H\left(\alpha_{j}\right)^{*}}{1-\left\langle\alpha_{i}, \alpha_{j}\right\rangle}\right]
$$

are positive semidefinite for all finite subsets $\left\{\alpha_{j}: 1 \leq j \leq k\right\}$ of $\mathbb{B}_{n}$.

Another immediate consequence of Theorem 2.1 is the analogue of the Carathéodory Theorem. Every element $A$ of $\mathfrak{L}_{n}$ has a Fourier expansion $A \sim \sum_{w \in \mathcal{F}_{n}} a_{w} L_{w}$ determined by $A \xi_{1}=\sum_{w \in \mathcal{F}_{n}} a_{w} \xi_{w}$. The Cesaro means of this series converge weak-* to $A$ [9]. Similarly, we may define the Fourier expansion of elements of $\mathfrak{M}_{p}\left(\mathfrak{L}_{n}\right)$ with coefficients in $\mathfrak{M}_{p}$ : in this case write $A \sim \sum_{w \in \mathcal{F}_{n}} L_{w} \otimes a_{w}$, where $a_{w} \in \mathfrak{M}_{p}$. The Carathéodory problem specifies an initial segment of this series and asks if it can be completed to an operator of norm at most one. Let a subset $S$ of $\mathcal{F}_{n}$ be called a (left) initial segment if it has the property that whenever $w=u v$ belongs to $S$, then so does $u$. Let $P_{S}$ denote the orthogonal projection onto $\operatorname{span}\left\{\xi_{w}: w \in S\right\}$. The initial segment property ensures that $P_{S}$ is invariant under $\mathfrak{R}_{n}^{*}$ and hence $P_{S}^{\perp}$ belongs to $\operatorname{Lat}\left(\Re_{n}\right)$.

THEOREM 3.6. Suppose that $S$ is a finite left initial segment of $\mathcal{F}_{n}$ and let $C_{w}$ be given matrices in $\mathfrak{M}_{p}$ for $w \in S$. Then there is an element $A$ in the unit ball of $\mathfrak{M}_{p}\left(\mathfrak{L}_{n}\right)$ with Fourier coefficients $a_{w}=C_{w}$ for $w \in S$ if and only if

$$
\left\|\left(P_{S} \otimes I_{p}\right)\left(\sum_{w \in S} L_{w} \otimes C_{w}\right)\right\| \leq 1
$$

We remark that finiteness of $S$ is not necessary, although one then needs to make sense of the sum over $S$ in the Cesaro sense.

Proof. The set of elements of $\mathfrak{L}_{n}$ interpolating the zero data $C_{w}=0_{1}$ for all $w \in S$ is the WOT-closed right ideal $\mathfrak{J}$ with $\mu(\mathfrak{J})=P_{S}^{\perp} \mathcal{H}_{n}$. Thus as before, given data $C_{w}$, the desired element exists if and only if the distance from $\sum_{w \in S} L_{w} \otimes C_{w}$ to $\mathfrak{M}_{p}(\mathfrak{J})$ is at most one. The result now follows from the distance formula.

An natural example of this phenomenon is the set of all words of length at most $k$. In this case, the ideal is

$$
\mathfrak{L}_{n}^{0, k}:=\left\{A \in \mathfrak{L}_{n}: A=\sum_{\substack{w \in \mathcal{F}_{n} \\|w|>k}} a_{w} L_{w}\right\}
$$


which is two sided. The projection $P_{k}$ onto $\operatorname{span}\left\{\xi_{w}:|w| \leq k\right\}$ is co-invariant and thus $P_{k} A=P_{k} A P_{k}$ for all $A$ in $\mathfrak{L}_{n}$. Hence we obtain a new proof of a result of Popescu [21, Corollary 4.2]:

COROLLARY 3.7. Given a finite power series $\sum_{|w| \leq k} a_{w} w$ belonging to the semigroup algebra $\mathbb{C} \mathcal{F}_{n}$, there is a element $A \in \mathfrak{L}_{n}$ with $\|A\| \leq 1$ and $A-\sum_{|w| \leq k} a_{w} L_{w} \in \mathfrak{L}_{n}^{0, k}$ if and only if $\left\|P_{k} \sum_{|w| \leq k} a_{w} L_{w}\right\| \leq 1$.

QUESTION 3.8. When this occurs, is there an isometry in the coset? More concretely, if $A \in \mathfrak{L}_{n}$ is norm one and achieves its norm, is it necessarily an isometry?

This question is motivated by the fact that this situation is obtained when $n=1$. Moreover, the interpolating function may be taken to be a finite Blaschke product of degree at most $k$. However the operator-theoretic argument usually used in that case does not extend directly to the non-commutative setting. Partial results are readily obtained which indicate that it may well be true nevertheless.

\section{REPRODUCING KERNEL HILBERT SPACES}

In this section, we wish to point out a more function theoretic viewpoint on this material which relates our interpolation results more closely to other similar results in the literature. In particular, we outline a proof that the symmetric Fock space is an analytic reproducing kernel Hilbert space, and connect the interpolation results here to interpolation results of Agler [2], McCullough [14] and Quiggin [24] in similar settings, and to commutative dilation results of Popescu [22] and Arveson [5].

Specifically, we are concerned with Nevanlinna-Pick interpolation. In this case, the ideal in question always contains the commutator ideal $\overline{\mathfrak{C}}$, as it is the kernel of the Gelfand map into $H^{\infty}\left(\mathbb{B}_{n}\right)$. The orthogonal complement of the range of $\overline{\mathfrak{C}}$ is $\mathcal{H}_{n}^{s}$, the symmetric Fock space. Let $P_{s}$ denote the projection of $\mathcal{H}_{n}$ onto $\mathcal{H}_{n}^{s}$. The compressions $S_{i}=\left.P_{s} L_{i}\right|_{\mathcal{H}_{n}^{s}}$ generate a WOT-closed algebra $\mathfrak{S}$ which is completely isometrically isomorphic to $\mathfrak{L}_{n} / \overline{\mathfrak{C}}$ by Corollary 2.3.

Arveson [5] shows that the symmetric Fock space and these operators $S_{i}$ form an analytic reproducing kernel Hilbert space. We briefly outline this from our viewpoint. Let $\mathbb{N}_{0}$ 
be the set of non-negative integers. For each $\lambda \in \mathbb{B}_{n}$ and each $n$-tuple $k=\left(k_{1}, \ldots, k_{n}\right) \in \mathbb{N}_{0}^{n}$, let $\lambda^{k}:=\lambda_{1}^{k_{1}} \ldots \lambda_{n}^{k_{n}}$. For $k \in \mathbb{N}_{0}^{n}$, let $\mathcal{P}_{k}:=\left\{w \in \mathcal{F}_{n}: w(\lambda)=\lambda^{k}\right.$ for all $\left.\lambda \in \mathbb{B}_{n}\right\}$. Define the vector

$$
\zeta^{k}:=\frac{1}{\left|\mathcal{P}_{k}\right|} \sum_{w \in \mathcal{P}_{k}} \xi_{w}
$$

Clearly this is a set of symmetric words which also spans $\mathcal{H}_{n}^{s}$, are pairwise orthogonal, and $\left\|\zeta^{k}\right\|=\left|\mathcal{P}_{k}\right|^{-1 / 2}$. Note that

$$
\left|\mathcal{P}_{k}\right|=\left(\begin{array}{c}
|k| ! \\
k_{1} ! k_{2} ! \cdots k_{n} !
\end{array}\right)
$$

This space is spanned by the kernel vectors $\left\{\nu_{\lambda}: \lambda \in \mathbb{B}_{n}\right\}$ by [10, Proposition 2.4], where

$$
\nu_{\lambda}=\left(1-\|\lambda\|^{2}\right)^{1 / 2} \sum_{w \in \mathcal{F}_{n}} \overline{w(\lambda)} \xi_{w}=\left(1-\|\lambda\|^{2}\right)^{1 / 2} \sum_{k \in \mathbb{N}_{0}^{n}} \bar{\lambda}^{k}\left|\mathcal{P}_{k}\right| \zeta^{k}
$$

Set

$$
u_{\lambda}=\left(1-\|\lambda\|^{2}\right)^{-1 / 2} \nu_{\lambda}=\sum_{k \in \mathbb{N}_{0}^{n}} \bar{\lambda}^{k}\left|\mathcal{P}_{k}\right| \zeta^{k} .
$$

Note that for each $\lambda \in \mathbb{B}_{n}$,

$$
\left\langle\zeta^{k}, u_{\lambda}\right\rangle=\lambda^{k}\left|\mathcal{P}_{k}\right|\left\|\zeta^{k}\right\|^{2}=\lambda^{k}
$$

Thus every vector $\zeta=\sum_{k \in \mathbb{N}_{0}^{n}} c_{k} \zeta^{k}$ in $\mathcal{H}_{n}^{s}$ has a functional representation on $\mathbb{B}_{n}$ given by

$$
\zeta(\lambda)=\left\langle\zeta, u_{\lambda}\right\rangle=\sum_{k \in \mathbb{N}_{0}^{n}} c_{k} \lambda^{k}
$$

Moreover

$$
|\zeta(\lambda)| \leq\|\zeta\|\left\|u_{\lambda}\right\|=\|\zeta\|\left(1-\|\lambda\|^{2}\right)^{-1 / 2}
$$

Thus $\mathcal{H}_{n}^{s}$ becomes a Hilbert space of analytic functions in which the point evaluations are continuous. To emphasize that this is an $L^{2}$ norm on these functions, we will write $\|f\|_{2}$ for the norm of an element $f$ in $\mathcal{H}_{n}^{s}$.

The important fact is that the operators $S_{i}$ have a nice analytic form in this representation. Indeed, if $A$ is any operator in $\mathfrak{L}_{n}$ and $S=\left.P_{s} A\right|_{\mathcal{H}_{n}^{s}}$ is the compression, then because $A^{*} \nu_{\lambda}=\overline{\hat{A}(\lambda)}$,

$$
S \zeta(\lambda)=\left\langle A \zeta, u_{\lambda}\right\rangle=\left\langle\zeta, A^{*} u_{\lambda}\right\rangle=\hat{A}(\lambda)\left\langle\zeta, u_{\lambda}\right\rangle=\hat{A}(\lambda) \zeta(\lambda)
$$


Thus the operators in $\mathfrak{S}$ are analytic multipliers on $\mathcal{H}_{n}^{s}$, and we may write $S=M_{h}$ where $h=\hat{A}$. Thus the operator norm equals the multiplier norm:

$$
\|S\|=\left\|M_{h}\right\|=\sup \left\{\|h f\|_{2}:\|f\|_{2} \leq 1\right\} .
$$

In particular, $S_{i}=M_{\lambda_{i}}$ are the multipliers by the coordinate functions.

Conversely, suppose that $h$ is a bounded multiplier; so that $M_{h}$ is a bounded operator. Then the Cesaro means $h_{n}=C_{n}(f)$ are polynomials and $M_{h_{n}}$ converges to $M_{h}$ in the strong operator topology (cf. [9, Lemma 1.1]). Since each $M_{h_{n}}$ is a polynomial in the $S_{i}$ 's, it follows that $M_{h}$ belongs to $\mathfrak{S}$. Thus $\mathfrak{S}$ is precisely the algebra of multipliers.

Thus Theorem 3.3 may be reformulated as:

COROLLARY 4.1. An element $h \in H^{\infty}\left(\mathbb{B}_{n}\right)$ determines a multiplier $M_{h}$ of norm at most one if and only if the matrices

$$
\left[\frac{1-h\left(\lambda_{i}\right) \overline{h\left(\lambda_{j}\right)}}{1-\left\langle\lambda_{i}, \lambda_{j}\right\rangle}\right]
$$

are positive for all finite subsets $\left\{\lambda_{j}: 1 \leq i, j \leq k\right\}$ of $\mathbb{B}_{n}$.

Similarly, Theorem 3.2, Corollary 3.5 and Corollary 3.7 may be recast as statements about matrix multipliers. This formulation suggests a related result in the same spirit, namely a Caratheodory result for multipliers. Every multiplier $h$ has a power series expansion

$$
h(\lambda)=\sum_{k \in \mathbb{N}_{0}^{n}} h_{k} \lambda^{k}
$$

determined by

$$
M_{h} \zeta^{0}=\sum_{k \in \mathbb{N}_{0}^{n}} h_{k} \zeta^{k}
$$

Let $\mathcal{S}_{p}=\operatorname{span}\left\{\zeta^{k}:|k| \leq p\right\}$.

THEOREM 4.2. Given a finite power series $\sum_{|k| \leq p} a_{k} \zeta^{k}$, there is a multiplier $M_{h}$ with $\left\|M_{h}\right\| \leq 1$ such that $h_{k}=a_{k}$ for $|k| \leq p$ if and only if $\left\|P_{\mathcal{S}_{p}} \sum_{|k| \leq p} a_{k} M_{z^{k}}\right\| \leq 1$.

Proof. The norm condition is the compression of any solution $M_{h}$ to $\mathcal{S}_{p}$, and thus the norm condition is necessary. For the converse, consider the ideal $\mathfrak{J}=\overline{\mathfrak{C}}+\mathfrak{L}_{n}^{0, p}$ of $\mathfrak{L}_{n}$. 
This is the WOT-closed two-sided ideal such that $\left(\mathfrak{J} \mathcal{H}_{n}\right)^{\perp}=\mathcal{S}_{p}$. By Theorem 2.1, there is an element $A$ in $\mathfrak{L}_{n}$ with $\|A\| \leq 1$ such that

$$
\widehat{A}(\lambda)=\sum_{|k| \leq p} a_{k} \lambda^{k}+\text { higher order terms. }
$$

The compression of $A$ to $\mathcal{H}_{n}^{s}$ is a multiplier $M_{h}$ with the desired properties.

The vectors $u_{\lambda}$ are the reproducing kernel functions. They determine a positive definite function on $\mathbb{B}_{n} \times \mathbb{B}_{n}$ given by

$$
K(\lambda, \mu)=\left\langle u_{\mu}, u_{\lambda}\right\rangle=\sum_{k \in \mathbb{N}_{0}^{n}}\left(\begin{array}{c}
|k| ! \\
k_{1} ! k_{2} ! \cdots k_{n} !
\end{array}\right) \lambda^{k} \bar{\mu}^{k}=\frac{1}{1-\langle\lambda, \mu\rangle} .
$$

Agler [2] reformulated the classical Nevanlinna-Pick problem to focus on the role of the kernel function, and showed that the Sobolev space of $L^{2}$-differentiable functions on $(0,1)$ also satisfies the corresponding interpolation theorem. This idea is pursued by McCullough [14] and Quiggin [24]. In particular, Quiggin defines a kernel function $k(x, y)$ on $X^{2}$ to be an NP kernel provided that whenever $E$ is a (finite) subset of $X$ and $f$ is a function on $E$, there is an extension of $f$ to a function $F$ on $X$ such that the multiplier $\left\|M_{F}\right\| \leq 1$ if and only if the kernel

$$
\left(1-f f^{*}\right) K(x, y)=[K(x, y)(1-\overline{f(x)} f(y))] \quad \text { for } \quad x, y \in E
$$

is positive. Moreover, he establishes a useful sufficient condition to guarantee that this occurs: if $K(x, y) \neq 0$ on $X^{2}$ and the function $\left.(1 / K)\right|_{E}$ has exactly one positive eigenvalue for all finite subsets $E$ of $X$, then $K$ is an NP kernel. McCullough shows that this condition is also sufficient to imply it is a complete NP kernel, meaning that the matrix version of the Nevanlinna-Pick theorem is valid.

In our case, the reciprocal of the kernel function is $1-\langle\lambda, \mu\rangle$ which for a subset $E=\left\{\lambda_{1}, \ldots, \lambda_{k}\right\}$ leads to the matrices $\mathbf{1} \cdot \mathbf{1}^{*}-\Lambda^{*} \Lambda$, where $\mathbf{1}$ is the column vector consisting of $k 1$ 's, and $\Lambda=\left(\lambda_{1}, \ldots, \lambda_{k}\right)$ is an $n \times k$ matrix with columns equal to $\lambda_{i}$. Now $\Lambda^{*} \Lambda$ is positive and norm less than $k$; while $\mathbf{1} \cdot \mathbf{1}^{*}$ is rank one and norm exactly $k$. It follows that $\mathbf{1} \cdot \mathbf{1}^{*}-\Lambda^{*} \Lambda$ has exactly one positive eigenvalue. So this is an NP kernel by Quiggin's Theorem, which leads to a rather different proof than ours.

We turn now to the connection with dilation theory mentioned above. The connection comes from the fact that the $n$-tuple $\left(S_{1}, \ldots, S_{n}\right)$ is a model for commuting $n$-tuples 
$T=\left(T_{1}, \ldots, T_{n}\right)$ satisfying

$$
T T^{*}=T_{1} T_{1}^{*}+\cdots+T_{n} T_{n}^{*} \leq I
$$

This condition is merely that $\|T\| \leq 1$ as an operator from $\mathcal{H}^{(n)}$, the sum of $n$ copies of Hilbert space $\mathcal{H}$, into $\mathcal{H}$. The main dilation result in this context is the following result. It appears in Popescu [22, Theorem 9.2] and Arveson [5, Theorem 6.3]. We provide another proof which shows how it follows from the non-commutative dilation theorem of Bunce [8] and Frahzo [12].

THEOREM 4.3. Let $T=\left(T_{1}, \ldots, T_{n}\right)$ be an $n$-tuple of operators on a Hilbert space $\mathcal{H}$ such that $\|T\| \leq 1$. Then there is a (unique) completely positive map $\Phi$ from $\mathrm{C}^{*}\left(S_{1}, \ldots, S_{n}\right)$ into $\mathcal{B}(\mathcal{H})$ such that

$$
\Phi\left(p(S) q\left(S^{*}\right)\right)=p(T) q\left(T^{*}\right)
$$

for all polynomials $p$ and $q$ in $n$ commuting variables.

Proof. Fix $r<1$ and consider $r T=\left(r T_{1}, \ldots, r T_{n}\right)$. Then by Proposition 2 of [8], there exists a Hilbert space $\mathcal{K} \supseteq \mathcal{H}$ and pure isometries $C_{1}, \ldots, C_{n}$ on $\mathcal{K}$ with pairwise orthogonal ranges such that $\mathcal{H}$ is invariant for $C_{i}^{*}$ and $\left.C_{i}^{*}\right|_{\mathcal{H}}=r T_{i}^{*}$ for all $i$. For any operator $A$, let $A^{(\infty)}$ be the direct sum of $A$ with itself countably many times. Since the $C_{i}$ are pure isometries, it follows that the $n$-tuples $\left(C_{1}^{(\infty)}, \ldots, C_{n}^{(\infty)}\right)$ and $\left(L_{1}^{(\infty)}, \ldots, L_{n}^{(\infty)}\right)$ are unitarily equivalent. Therefore, there is an $\mathfrak{L}_{n}^{(\infty)}$-invariant subspace $\mathcal{M}$ of $\mathcal{H}_{n}^{(\infty)}$ such that

$$
\left.r T_{i}^{*} \simeq L_{i}^{*(\infty)}\right|_{\mathcal{M}^{\perp}} \quad \text { for } \quad 1 \leq i \leq n
$$

Thus $\left.r T_{i} \simeq P_{\mathcal{M}^{\perp}}^{\perp} L_{i}^{(\infty)}\right|_{\mathcal{M}^{\perp}}$. There is a canonical $*$-isomorphism of the $\mathrm{C}^{*}$-algebra $\mathrm{C}^{*}\left(L_{1}, \ldots, L_{n}\right)$ into $\mathcal{B}\left(\mathcal{H}_{n}^{(\infty)}\right)$ taking each $A$ to $A^{(\infty)}$. Define $\Psi_{r}(A)$ to be the compression $\left.P_{\mathcal{M}}^{\perp} A^{(\infty)}\right|_{\mathcal{M}^{\perp}}$ of $A^{(\infty)}$ to $\mathcal{M}^{\perp}$ followed by the unitary equivalence which identifies $\mathcal{M}^{\perp}$ with $\mathcal{H}$. This is completely positive, contractive and satisfies

$$
\begin{aligned}
\Psi_{r}\left(p(L) q\left(L^{*}\right)\right) & \left.\simeq P_{\mathcal{M}}^{\perp} p(L)^{(\infty)} q\left(L^{*}\right)^{(\infty)}\right|_{\mathcal{M}^{\perp}} \\
& =\left.P_{\mathcal{M}}^{\perp} p(L)^{(\infty)} P_{\mathcal{M}}^{\perp} q\left(L^{*}\right)^{(\infty)}\right|_{\mathcal{M}^{\perp}} \simeq p(r T) q\left(r T^{*}\right) .
\end{aligned}
$$

Now let $r$ increase to 1 to obtain a completely positive contractive map $\Psi$ such that $\Psi\left(p(L) q\left(L^{*}\right)\right)=p(T) q\left(T^{*}\right)$. 
Now notice that $\mathcal{M}^{\perp}$ must be contained in $\mathcal{H}_{n}^{s(\infty)}$. Indeed, one sees that for any words $u, v \in \mathcal{F}_{n}$,

$$
0=[u(T), v(T)]^{*} x \simeq\left[L_{u}^{(\infty)}, L_{v}^{(\infty)}\right]^{*} \xi
$$

for all vectors $\xi \in \mathcal{M}^{\perp}$. Expressing each vector in $\mathcal{H}_{n}^{(\infty)}$ as $\xi=\sum_{w \in \mathcal{F}_{n}} x_{w} \otimes \xi_{w}$ (where the coefficients $x_{w}$ are vectors in $\ell^{2}$ ), one readily obtains that $x_{u v w}=x_{v u w}$ for all words $u, v, w \in \mathcal{F}_{n}$. This establishes our claim.

Hence it follows that $\Psi$ factors through compression to $\mathcal{H}_{n}^{s}$, followed by the desired completely contractive $\Psi$ of $\mathrm{C}^{*}\left(S_{1}, \ldots, S_{n}\right)$ into $\mathcal{B}(\mathcal{H})$ with the required properties.

The connection between dilations and interpolation comes via the commutant lifting theorem. This appraoch was pioneered by Sarason [25] who proved the commutant lifting theorem for parts of the shift prior to the general formulation by Sz. Nagy and Foiaş [26]. In the present context, Popescu established a version of the Caratheodory interpolation using a commutant lifting theorem for $n$-tuples of contractions which dilate to a multiple of the non-commuting shifts $L_{i}$. However, his theorem yields a commutative commutant lifting theorem which fits into the context of the present discussion and provides an alternate route to some of these results. We do not attempt to obtain the most general formulation, but rather try to illustrate the connection between these dilation results and our interpolation results.

PROPOSITION 4.4. Let $\mathcal{M}^{\perp}$ be an invariant subspace of the algebra $\mathfrak{S}^{(p)}$ acting on $\mathcal{H}_{n}^{s(p)}$, and let $T_{i}=\left.P_{\mathcal{M}} S_{i}^{(p)}\right|_{\mathcal{M}}$. Suppose that $X$ is a contraction in $\mathcal{B}(\mathcal{M})$ commuting with each $T_{i}$ for $1 \leq i \leq n$. Then there is a contraction $Y$ in $\mathfrak{M}_{p}(\mathfrak{S})$ such that $X=\left.P_{\mathcal{M}} Y\right|_{\mathcal{M}}$.

Proof. Since each $S_{i}$ is the compression of $L_{i}$ to $\mathcal{H}_{n}^{s}$, it follows equally well that the $T_{i}$ 's are compressions of $L_{i}^{(p)}$ 's. Therefore by Popescu's commutant lifting theorem [18], there is a contraction $Z$ in the commutant of $\mathfrak{L}_{n}^{(p)}$ such that $X=\left.P_{\mathcal{M}} Z\right|_{\mathcal{M}}$. Now this commutant equals $\mathfrak{M}_{p}\left(\mathfrak{R}_{n}\right)$. Let $Y$ be the compression of $Z$ to $\mathcal{H}_{n}^{s(p)}$. This is the desired operator because the left and right creation operators $L_{i}$ and $R_{i}$ on Fock space have the same compression to $\mathcal{H}_{n}^{s}$. Hence $Y$ belongs to $\mathfrak{M}_{p}(\mathfrak{S})$, which is the commutant of $\mathfrak{S}^{(p)}$.

Now for example, one may obtain a multiplier version of Nevanlinna-Pick interpolation. Of course, this result is an immediate corollary of our Theorem 3.2 and the discussion on reproducing kernels. So we just sketch the proof. 
COROLLARY 4.5. Given $k$ distinct points $\alpha_{j}$ in $\mathbb{B}_{n}$ and matrices $C_{j}$ in $\mathfrak{M}_{p}$ for $1 \leq j \leq k$, there is a matrix multiplier $M_{H}$ in the unit ball of $\mathfrak{M}_{p}\left(\mathfrak{S}_{n}\right)$ such that $H\left(\alpha_{j}\right)=C_{j}$ for $1 \leq j \leq k$ if and only if the matrix

$$
\left[\frac{I_{p}-C_{i} C_{j}^{*}}{1-\left\langle\alpha_{i}, \alpha_{j}\right\rangle}\right]_{k \times k}
$$

is positive semidefinite.

Proof. Consider the subspace $\mathcal{S}=\operatorname{span}\left\{u_{\alpha_{j}}: 1 \leq j \leq k\right\}$. Let $\mathcal{M}=\mathcal{S}^{(p)}$, and define an operator $X$ on $\mathcal{M}$ by setting the restriction of $X^{*}$ to the subspace $u_{\alpha_{j}} \otimes \mathbb{C}^{p}$ to be equal to $C_{j}^{*}$. It is routine to verify that $X^{*}$ commutes with the restrictions $T_{i}^{*}$ of $S_{i}^{*(p)}$ to $\mathcal{M}$, because these operators act on each $u_{\alpha_{j}} \otimes \mathbb{C}^{p}$ as the scalar operators $\left(\overline{\alpha_{j}}\right)_{i} I_{p}$. A calculation shows that the matrix condition in our hypothesis is equivalent to $X X^{*} \leq I$. Consequently, this condition is necessary for the existence of $H$. Conversely, by Proposition 4.4, $X$ dilates to a contractive multiplier $M_{H}$. It is evident that $H\left(\alpha_{j}\right)=C_{j}$ for $1 \leq j \leq k$ as desired.

\section{REFERENCES}

[1] G. Adams, P. McGuire and V. Paulsen, Analytic reproducing kernels and multiplication operators, Illinois J. Math. 36 (1992), 404-419.

[2] J. Agler, Nevanlinna-Pick interpolation on Sobolev space, Proc. Amer. Math. Soc. 108 (1990), 341-351.

[3] A. Arias and G. Popescu, Factorization and reflexivity on Fock spaces, Int. Equat. Oper. Theory 23 (1995), 268-286.

[4] A. Arias and G. Popescu, Noncommutative interpolation and Poisson transforms, preprint, 1997.

[5] W. Arveson, Subalgebras of $C^{*}$-algebras III: multivariable operator theory, preprint, 1997.

[6] H. Bercovici, C. Foiaş and C. Pearcy, Dual algebras with applications to invariant subspaces and dilation theory, CBMS Notes 56, Amer. Math. Soc., Providence, 1985.

[7] H. Bercovici, Hyper-reflexivity and the factorization of linear functionals, preprint, 1997.

[8] J. Bunce, Models for n-tuples of non-commuting operators, J. Func. Anal. 57 (1984), 21-30.

[9] K.R. Davidson and D.R. Pitts, Invariant subspaces and hyper-reflexivity for free semigroup algebras, preprint, 1996.

[10] K.R. Davidson and D.R. Pitts, The Algebraic Structure of Non-commutative Analytic Toeplitz Algebras, Math. Ann., to appear.

[11] C. Foiaş and A. Frahzo, The commutant lifting approach to interpolation problems, Operator Theory: Adv. and Appl. 44, Birhauser Verlag, Basel, 1990.

[12] A. Frahzo, Models for non-commuting operators, J. Func. Anal. 48 (1982), 1-11. 
[13] A. Frahzo, Complements to models for non-commuting operators, J. Func. Anal. 59 (1984), 445-461.

[14] S. McCullough, The local de Branges-Rovnyak construction and complete Nevanlinna-Pick kernels, Algebraic methods in operator theory, 15-24, Birkhauser Boston, Boston, MA, 1994.

[15] V.I. Paulsen, Completely bounded maps and dilations, Pitman Res. Notes Math. 146, Longman Sci. Tech. Harlow, 1986.

[16] G. Popescu, Isometric dilations for infinite sequences of noncommuting operators, Trans. Amer. Math. Soc. 316 (1989), 523-536.

[17] Popescu, G., Characteristic functions for infinite sequences of noncommuting operators, J. Operator Thy. 22 (1989), 51-71.

[18] G. Popescu, Multi-analytic operators and some factorization theorems, Indiana Univ. Math. J. 38 (1989), 693-710.

[19] G. Popescu, Von Neumann Inequality for $\left(\mathcal{B}(\mathcal{H})^{n}\right)_{1}$, Math. Scand. 68 (1991), 292-304.

[20] G. Popescu, Functional calculus for noncommuting operators, Mich. J. Math. 42 (1995), 345-356.

[21] G. Popescu, Multi-analytic operators on Fock spaces, Math. Ann. 303 (1995), 31-46.

$[22]$ G. Popescu, Poisson transforms on some $C^{*}$-algebras generated by isometries, preprint, 1995.

[23] G. Popescu, Noncommuting disc algebras and their representations, Proc. Amer. Math. Soc. 124 (1996), $2137-2148$.

[24] P. Quiggen, For which reproducing kernel Hilbert spaces is Pick's theorem true? Integr. Equat. Oper. Th. 16 (1993), 244-266.

[25] D. Sarason, Generalized interpolation in $H^{\infty}$, Trans. Amer. Math. Soc. 127 (1967), 179-203.

[26] B. Sz. Nagy and C. Foiaş, Harmonic analysis of operators on Hilbert space, North Holland Pub. Co., London, 1970.

Pure Math. Dept., U. Waterloo, Waterloo, ON N2L-3G1, CANADA

E-mail address: krdavidson@math.uwaterloo.ca

Math. Dept., University of Nebraska, Lincoln, NE 68588, USA

E-mail address: dpitts@math.unl.edu 